\title{
AVALIAÇÃO DA EXTRAÇÃO DE ÓLEO DE SOJA COM ETANOL
}

\author{
Eduardo Henrique Rotta ${ }^{1}$, Letiane Thomas Hendges ${ }^{2}$, Júlia Bitencourt \\ Welter $^{3}$ e Bruno München Wenzel ${ }^{4}$
}

\begin{abstract}
Resumo: A sensibilização para questões energéticas e ambientais decorrentes da queima de combustíveis fósseis têm incentivado a busca por combustíveis renováveis, entre eles o biodiesel. Industrialmente, ele é produzido via reação de transesterificação de um óleo previamente extraído da oleaginosa (usualmente o hexano como solvente), e um álcool. Neste contexto torna-se importante avaliar a possibilidade de utilização do etanol como agente de extração, já que também participa da reação, podendo assim simplificar o processo. Neste trabalho, com base em um planejamento experimental, foi avaliada a eficiência de extração de óleo em sementes de soja, no equilíbrio, utilizando etanol como solvente, em função das seguintes variáveis: temperatura (entre 30 e $70^{\circ} \mathrm{C}$ ), razão solvente:óleo (10 a $20 \mathrm{~g}$ de etanol/g de óleo) e pureza do etanol (95,5 à 99,5 wt\%). Utilizaram-se grãos de soja com teor de umidade e voláteis de 5,71 wt\% e teor de óleo de 18,31 wt\%. Os experimentos apresentaram extração entre 35 e 100\%. Uma análise de variância apontou que ambas as variáveis investigadas influenciam direta e significativamente o equilíbrio da extração. Um modelo linear de primeira ordem mostrou-se suficiente para predição dos resultados experimentais e foi utilizado para calcular valores das variáveis que mostraram elevadas eficiências de extração.
\end{abstract}

Palavras-chave: Extração sólido-líquido. Etanol. Óleo de soja. Planejamento experimental.

\section{Introdução}

A energia tornou-se um fator crucial para a humanidade manter $\mathrm{o}$ atual crescimento econômico e alto padrão de vida. Estima-se que o mundo precisará de $46 \%$ mais energia em 2040 do que em 2010 , sendo que o setor de transportes, em específico, deverá aumentar sua demanda por energia em cerca de $40 \%$ neste mesmo período (U. S. ENERGY INFORMATION ADMINISTRATION, 2016). Mundialmente, acredita-se que este setor é responsável por cerca de $60 \%$ da demanda de petróleo e será $O$ setor de maior crescimento da demanda de energia no futuro (ATABANI et al., 2012). Paralelamente, os dados do Painel Intergovernamental de Mudanças Climáticas das Nações Unidas (IPCC, 2014) apontam para mudanças climáticas relacionadas à queima de combustíveis fósseis, pela emissão de gases de efeito estufa. A sensibilização para as questões energéticas e ambientais decorrentes da queima de combustíveis fósseis tem incentivado a busca por fontes alternativas de energia - fontes renováveis ao invés do petróleo e seus derivados. Entre essas fontes, no que tange o setor de transportes, destacam-se o etanol e o biodiesel (ATABANI et al., 2012).

O biodiesel é produzido a partir da transesterificação de óleos vegetais ou gorduras animais com um álcool de cadeia curta e o uso de um catalisador (KNOTHE et al., 2005; HINCAPIÉ; MONDRAGÓN; LÓPEZ, 2011). Segundo relatório da Rede de Políticas Energéticas Renováveis para o Século XXI (REN21, 2015), a produção de biodiesel é baseada largamente na utilização de óleos vegetais, destacando-se o óleo de colza na Europa e o óleo de soja nos EUA, Brasil e Argentina. No Brasil, de acordo com dados da Empresa de Pesquisa Energética (2016), o montante de biodiesel produzido em 2015 foi de $3.937 .269 \mathrm{~m}^{3}$, sendo que $70 \%$ foi produzido a partir do óleo extraído da soja, maior cultura sazonal do país. Assim, considerando a produção de biodiesel a partir de oleaginosas, exige-se que o óleo seja previamente extraído das sementes utilizando um solvente, sendo o hexano o mais comum (GANDHI et al., 2003; KARTIKA et al., 2016).

A utilização do hexano como solvente atende uma série de características

\footnotetext{
${ }^{1}$ E-mail: eh.rotta@gmail.com

${ }^{2}$ E-mail: letiane.hendges@hotmail.com

${ }^{3}$ E-mail: juliabwelter@gmail.com
}

${ }^{4}$ E-mail: bruno.wenzel@uffs.edu.br

Universidade Federal da Fronteira Sul - UFFS. Rua Jacob Reinaldo Haupenthal, 1580, 97900-000, Cerro Largo, Rio Grande do Sul, Brasil. 
REA - Revista de estudos ambientais (Online)

v.19, n. 1, p.27-35, jan./jun. 2017

determinantes para o processo de extração boa dissolução em óleo, composição homogênea, estreita faixa de temperatura de ebulição, imiscibilidade em água e baixo calor latente de ebulição -, obtendo-se elevadas eficiências de extração (MANDARINO; ROESSING, 2001; KNOTHE et al., 2005). Entretanto, de acordo com Li et al. (2014), algumas desvantagens, como não-renovável, inflamável, alta volatilidade e toxicidade (SAWADA et al., 2014; BAÜMLER; CARRÍN; CARELLI, 2016), reforçam a busca por outros solventes economicamente viáveis. Diversos têm sido estudados para extração de triacilgliceróis de diferentes matrizes oleaginosas, como estudos a respeito da utilização de álcoois como o metanol, etanol e isopropanol (DAGOSTIN; CARPINÉ; CORAZZA, 2015; TUNTIWIWATTANAPUN; TONGCUMPOU; WIESENBORN, 2016), além de fluidos subcríticos e supercríticos (ANG et al., 2015; FERNÁNDEZ et al., 2015; RAl; MOHANTY; BHARGAVA, 2016).

No contexto da produção de biodiesel, a utilização do etanol se destaca pelo fato de que, além de ser empregado como agente de extração, pode ser utilizado como reagente no processo de transesterificação, diminuindo etapas de separação e/ou purificação do óleo e possibilitando a integração dos processos de extração e transesterificação (HINCAPIÉ; MONDRAGÓN; LÓPEZ, 2011; SANGALETTI-GERHARD et al., 2014) Tratase ainda, de um solvente de baixo custo, com grande variedade de matérias-primas, resultando em mais de 97 milhões de metros cúbicos produzidos no ano de 2015 em todo mundo, com destaque aos Estados Unidos e ao Brasil, responsáveis por $85 \%$ de toda produção (FERREIRA-DIAS; VALENTE; ABREU, 2003; RENEWABLE FUELS ASSOCIATION, 2016).

Neste contexto, o presente trabalho visa, através de experimentos de equilíbrio, explorar a viabilidade técnica do emprego do etanol como solvente de extração de óleo de soja. Para isso, aplicou-se um planejamento experimental fatorial completo em dois níveis com ponto central, objetivando investigar o efeito das seguintes variáveis sobre a eficiência de extração: temperatura $(T$, entre 30 e $70^{\circ} \mathrm{C}$ ), razão solvente:óleo ( $R$, entre 10:1 e 20:1) e pureza do etanol ( $P$, entre 95,5 e $99,5 \%$ ). Os experimentos foram realizados em duplicata, exceto para o ponto central, realizado em triplicata. A partir dos resultados da extração percentual de óleo de soja (variável resposta), avaliou-se estatisticamente os fatores e suas interações de maior grau de significância, bem como o ajuste de um modelo linear de primeira ordem para a estimativa da eficiência de extração sob o efeito de variáveis de operação.

\section{Metodologia}

\subsection{Preparo e caracterização das sementes de soja e etanol}

Grãos de soja (Glycine max) foram limpos através da remoção de impurezas e imperfeitos, secos e triturados em moinho analítico de lâminas. Foram utilizadas como amostras as partículas de grãos de soja com tamanho na faixa entre 0,71 e $1,41 \mathrm{~mm}$ (entre peneiras com malhas mesh tyler $25 \mathrm{e}$ 14). A amostra foi caracterizada em relação ao teor de umidade e material volátil e teor de óleo presente no grão, em quadruplicata. A umidade e material volátil foi determinado por ensaio com a metodologia padrão American Oil Chemists' Society - AOCS Ac 2-41 (2009), a qual estabelece a determinação por gravimetria, com a manutenção da amostra em temperatura de $130^{\circ} \mathrm{C}$ durante 3 horas. A determinação do teor de óleo foi realizada em extrator de óleos e graxas (MA 044/08/50, Marconi) com éter de petróleo, através da metodologia AOCS Ac 3-44 (1997) e instruções contidas no manual do equipamento.

Como solvente do processo investigado foi utilizado etanol em diferentes purezas. As soluções foram preparadas por diluição com água destilada a partir de reagente de pureza analítica $(99,9 w t \%)$.

\subsection{Planejamento experimental fatorial completo $2^{\mathrm{k}}$}

Adotou-se um planejamento experimental fatorial completo em dois níveis $\left(2^{k=3}\right)$, com ponto central, para as variáveis temperatura $(T)$, razão mássica entre etanol e óleo $(R)$ e pureza do etanol $(P)$. A seleção dos valores dos fatores se deu por análise de literatura, conforme segue. Foi selecionada como variável de resposta a eficiência de extração no equilíbrio.

A temperatura influencia significativamente na variação da viscosidade do óleo e a solubilidade do soluto (THOMAS, 2003). Na medida em que 


\section{REA - Revista de estudos ambientais (Online) v.19, n. 1, p.27-35, jan./jun. 2017}

a temperatura aumenta, a viscosidade do óleo diminui, penetrando com mais facilidade nos poros das partículas e, portanto, aumentando a quantidade de óleo extraída (ZACHI, 2007). Quanto à solubilidade do óleo em etanol anidro e hidratado, Rao et al. (1955) mostraram que para o óleo de soja a solubilidade aumenta significativamente até uma temperatura limite (aproximadamente $70^{\circ} \mathrm{C}$ ), sendo próxima à temperatura de ebulição do solvente, e que também aumenta com o aumento da pureza do etanol.

Com relação ao solvente, o processo de extração é afetado pela sua quantidade e qualidade. Thomas (2003) afirma que a quantidade de solvente depende da composição do grão, ou seja, quanto maior o teor de fibra maior será a quantidade de solvente necessária. Razões elevadas podem provocar maior solubilidade da glicerina, dificultando a separação de fases (TOMAZIN JR., 2008). No que diz respeito à qualidade, refere-se à pureza do solvente. Para o caso do etanol, ele pode estar na condição anidra (livre de água) e hidratada (com concentrações de água variáveis), sendo que 95,5 corresponde, aproximadamente, ao teor do etanol combustível.

Com base nesta análise, os fatores selecionados, as suas unidades de medida e os seus valores - sendo $(-1)$ para o nível inferior, (0) para o ponto central e $(+1)$ para o nível superior - podem ser conferidos na Tabela 1.

Tabela 1 - Variáveis independentes: valores reais e os respectivos níveis

\begin{tabular}{|c|c|c|c|c|c|}
\hline \multirow{2}{*}{ Fator } & \multirow{2}{*}{ Unidade } & \multirow{2}{*}{ Símbolo } & \multicolumn{3}{|c|}{ Níveis } \\
\hline & & & -1 & 0 & +1 \\
\hline Temperatura & ${ }^{\circ} \mathrm{C}$ & $\mathrm{T}$ & 30 & 50 & 70 \\
\hline Razão etanol:óleo & (g etanol):(g de óleo) & $\mathrm{R}$ & $10: 1$ & $15: 1$ & $20: 1$ \\
\hline Pureza do solvente & wt $\%$ & $\mathrm{P}$ & 95,5 & 97,5 & 99,5 \\
\hline
\end{tabular}

\subsection{Procedimento experimental}

Os experimentos foram realizados em frascos Erlenmeyers vedados com rolhas de borracha antiácida em banho termostático com agitação recíproca (Dubnoff). Em experimentos preliminares, foi determinado o tempo necessário para que a extração atinja o equilíbrio: $120 \mathrm{~h}$ para os experimentos conduzidos a $30^{\circ} \mathrm{C}, 72 \mathrm{~h}$ para $50^{\circ} \mathrm{C}$ e 16 horas para $70^{\circ} \mathrm{C}$.

Com base na combinação de valores das variáveis independentes, selecionadas no planejamento experimental, primeiramente a temperatura de experimentação foi estabelecida no banho termostático. Em todos os ensaios foram utilizadas $10 \mathrm{~g}$ da amostra de soja previamente preparada. Conforme os valores das variáveis razão etanol:óleo e pureza do etanol, foram calculadas as massas de etanol anidro $(99,9 \%)$ e água destilada necessárias para 0 experimento. Essas quantidades foram adicionadas aos frascos Erlenmeyers, o qual foi inserido no banhomaria. Quando em equilíbrio térmico, as $10 \mathrm{~g}$ de amostra de soja foram adicionadas e o cronômetro acionado.

Após decorrido o tempo necessário para se atingir o equilíbrio, as amostras foram retiradas do banho e filtradas a vácuo com papel filtro quantitativo de $90 \mathrm{~mm}$. As tortas de filtração e os frascos Erlenmeyers empregados foram lavados com aproximadamente $100 \mathrm{~mL}$ de etanol para remoção de resquícios de óleo. As micelas obtidas com este procedimento foram submetidas a determinação da eficiência de extração, conforme segue.

Metodologia semelhante foi adotada por Freitas e Lago (2007) e Cavalcante, De Sousa e Hamawaki (2011).

\subsection{Determinação analítica da eficiência de extração}

A eficiência de extração foi calculada por gravimetria, com base na quantidade de óleo presente nas $10 \mathrm{~g}$ de amostra em relação à quantidade extraída. A quantidade extraída, por sua vez foi obtida a partir da micela proveniente dos experimentos.

Para isso, a micela foi submetida à um evaporador rotativo à $60{ }^{\circ} \mathrm{C}$ operando sob vácuo entre 600 e $720 \mathrm{mmHg}$ por cerca de 3 h até a completa evaporação do etanol. O balão de evaporação permaneceu por mais uma hora em uma estufa de secagem à $60{ }^{\circ} \mathrm{C}$ para evaporar eventuais traços de solvente e para secagem da vidraria. Após este procedimento a massa de óleo presente foi medida por meio de balança semianalítica. 


\section{REA - Revista de estudos ambientais (Online) v.19, n. 1, p.27-35, jan./jun. 2017}

\section{Resultados e discussões}

\subsection{Caracterização das amostras}

Os valores obtidos na determinação do teor de umidade e teor de óleo contido na semente estão dispostos na Tabela 2.

Industrialmente, definem-se como aceitáveis valores de umidade entre $8,00 \mathrm{e}$ $12,00 \%$ após o condicionamento do grão, entretanto valores abaixo dessa faixa podem contribuir para o reaproveitamento da micela devido a menor hidratação do solvente ao longo da extração (THOMAS, 2003; ZACHI, 2007; TOMAZIN JR., 2008; RODRIGUES, 2011; SAWADA et al., 2014). Para o teor de óleo, estudos realizados em diferentes cultivares de grão apresentam uma faixa de 13,50 a $23,95 \%$ de óleo em massa de soja (ALBRECHT et al., 2008; CAVALCANTE; DE SOUSA; HAMAWAKI, 2011). Sendo assim, pode-se afirmar que os valores obtidos na caracterização da amostra estão em consonância com as características da semente de soja reportadas em literatura.

Tabela 2 - Caracterização da matéria-prima a partir dos teores de óleo e de umidade

\begin{tabular}{cc}
\hline Parâmetro & Resultado (\%) \\
\hline Teor de umidade & $(5,27 \pm 0,21)$ \\
Teor de óleo & $(18,31 \pm 0,98)$ \\
\hline
\end{tabular}

\subsection{Análise estatística dos dados do planejamento experimental}

Os experimentos realizados a partir do planejamento experimental fatorial completo em dois níveis foram analisados estatisticamente quanto ao efeito das variáveis independentes e suas interações sobre a eficiência percentual de extração de óleo de soja. A partir do experimento no ponto central, foi analisada a presença de curvatura quadrática (MONTGOMERY; RUNGER, 2003) utilizando o software Matlab.

Os experimentos executados e seus respectivos resultados experimentais, bem como valores da variável de resposta preditos pelo modelo de superfície de resposta (que será apresentado mais adiante), estão dispostos na Tabela 3. Os resultados experimentais são expressos como valor médio, com intervalo de confiança de $95 \%$ a partir de corridas experimentais realizadas em duplicata, exceto para o ponto central, conduzido em triplicata.

Como pode ser observado na corrida 8, obteve-se uma eficiência de extração acima de $100 \%$. Resultados semelhantes foram reportados por Dagostin, Carpiné e Corazza (2015) utilizando etanol e misturas de ésteres alquilos como co-solvente, e por Baumler, Carrin e Carelli (2016), fazendo uso do etanol como solvente para extração de óleo de girassol. Conforme apontado pela literatura, este comportamento pode estar atrelado ao fato de o etanol possuir capacidade de extrair outros compostos presentes no grão, além dos lipídeos, como compostos não-polares (polifenóis, pigmentos e açúcares solúveis). Tendo em vista essa característica do etanol não ser seletivo quando utilizado como solvente de extração, convém denominar o conteúdo extraído como "material extraído".

Tabela 3 - Valores experimentais e preditos para a matriz codificada: corridas 1 a 8 correspondem ao planejamento fatorial completo $2^{3}$ e a corrida 9 , o ponto central

\begin{tabular}{c|ccc|ccc}
\hline \multirow{2}{*}{ Corrida } & \multicolumn{3}{|c|}{ Fatores codificados } & \multicolumn{3}{c}{ X- Extração de óleo (\%) } \\
\cline { 2 - 6 } & $\mathbf{T}$ & $\mathbf{R}$ & $\mathbf{P}$ & \multicolumn{2}{c}{ Experimental $^{*}$} & Predito $^{* *}$ \\
\hline 1 & -1 & -1 & -1 & $35,1 \pm 0,4$ & 27,8 \\
2 & -1 & -1 & 1 & $39,9 \pm 0,6$ & 48,9 \\
3 & -1 & 1 & -1 & $43,6 \pm 1,2$ & 48,5 \\
4 & -1 & 1 & 1 & $77,1 \pm 4,5$ & 69,9 \\
5 & 1 & -1 & -1 & $59,4 \pm 0,0$ & 86,0 \\
6 & 1 & -1 & 1 & $93,4 \pm 2,7$ & 86,0 \\
7 & 1 & 1 & -1 & $89,7 \pm \pm, 1$ & 107,1 \\
8 & 1 & 1 & 1 & $101,8 \pm \pm 2,7$ & 67,4 \\
9 & 0 & 0 & 0 & $67,0 \pm 3,7$ & \pm \\
\hline
\end{tabular}

* Intervalo de confiança de $\pm 95 \%$ em torno da média. A variância foi calculada considerando 2 repetições das corridas experimentais, exceto para o ponto central, quando 3 repetições foram efetuadas. ${ }^{* *}$ Resultado do valor predito pelo modelo ajustado no item subsequente. 


\section{REA - Revista de estudos ambientais (Online) v.19, n. 1, p.27-35, jan./jun. 2017}

O efeito, a interação entre as variáveis utilizadas no planejamento experimental e a presença de curvatura quadrática foram estudados através de análise de variância (ANOVA), conforme apresentado na Tabela 4. A determinação dos efeitos foi baseada nas corridas 1 a 8 (Tabela 3), enquanto que a curvatura quadrática foi analisada com base na comparação da interceptação com o resultado do ponto central. $O$ erro experimental foi obtido com base nas réplicas em todos os experimentos. Todos os fatores principais (temperatura $(T)$, razão solvente:óleo $(R)$ e pureza do solvente $(P))$ influenciam significativamente, enquanto que as interações entre os fatores não apresentaram efeitos significativos, exceto a interação de maior ordem. Como pode ser observado, ainda, os resultados apontam não haver presença de curvatura quadrática significativa, indicando que um modelo de superfície de resposta de forma linear em relação aos parâmetros e de primeira ordem pode ser considerado suficiente para descrição dos resultados da extração em função dos parâmetros investigados.

Tabela 4 - Análise de variância (ANOVA) dos fatores e suas interações no modelo fatorial completo $2^{3}$ e teste de curvatura quadrática

\begin{tabular}{cccccc}
\hline Fonte de variação & $\begin{array}{c}\text { Soma dos } \\
\text { quadrados }\end{array}$ & $\begin{array}{c}\text { Graus de } \\
\text { liberdade }\end{array}$ & F-valor $\left(\mathbf{1 0}^{-2}\right)$ & p-valor & Decisão* \\
\hline T & 5526,4 & 1 & 1335,7 & $<0,001$ & $\mathrm{~S}$ \\
$\mathrm{R}$ & $1.775,8$ & 1 & 429,0 & $<0,001$ & $\mathrm{~S}$ \\
$\mathrm{P}$ & 1778,7 & 1 & 429,7 & $<0,001$ & $\mathrm{~S}$ \\
T R & 11,9 & 1 & 2,87 & 0,121 & $\mathrm{NS}$ \\
T P & 15,0 & 1 & 3,62 & 0,086 & $\mathrm{NS}$ \\
R P & 11,3 & 1 & 2,73 & 0,130 & $\mathrm{NS}$ \\
T R P & 642,4 & 1 & 155,2 & $<0,001$ & $\mathrm{~S}$ \\
Curvatura quadrática & 0,56 & 1 & 0,136 & 0,720 & $\mathrm{NS}$ \\
pura & & 10 & & & \\
Erro Experimental & 41,40 & 10 & &
\end{tabular}

* Baseado em um nível de 95\% de significância (nível de significância, $\alpha=0.05$ ): S - significativo; NS - nãosignificativo.

\subsection{Modelo de superfície de resposta}

Com a utilização das variáveis que demonstraram significância para o experimento, propôs-se um modelo linear de primeira ordem (Equação 1). O modelo ilustra a dependência da variável resposta (X, eficiência de extração, \%) em relação aos fatores codificados. Todas as variáveis afetam positivamente $\mathrm{o}$ equilíbrio da extração. O modelo foi ajustado a partir dos dados experimentais por meio de regressão linear. O coeficiente de determinação obtido foi de $R^{2}=0,930$, podendo-se afirmar que o modelo predito apresenta uma boa correlação aos dados obtidos em laboratório.

$$
X=67,4+18,6 T+10,5 R+10,5 P-6,34 T R P
$$

Os valores obtidos através da aplicação da Equação 1 podem ser observados na Tabela 3 , dispostos com o objetivo de compará-los com os dados experimentais. A Figura 1a apresenta o comportamento dos dados obtidos experimentalmente versus o comportamento predito pelo modelo. Conforme pode ser visto na Figura 1b, nenhum dos dados ultrapassou $3 \%$ de diferença entre o resultado experimental e o predito, bem como os resíduos apresentam distribuição randômica.

Uma vez que o sistema experimental apresenta um comportamento linear, com coeficiente angular positivo, pode-se aferir que a condição de máxima eficiência ocorre nos níveis mais altos (+1) dos fatores 


\section{REA - Revista de estudos ambientais (Online) v.19, n. 1, p.27-35, jan./jun. 2017}

temperatura $(T)$, razão etanol:óleo $(R)$ e pureza do solvente $(P)$, resultando na extração completa do óleo da oleaginosa por meio da utilização de etanol como solvente.

Figura 1 - Comparação entre os dados experimentais e os valores preditos pelo modelo: a) extração experimental versus extração predita pelo modelo, b) distribuição dos resíduos

a)

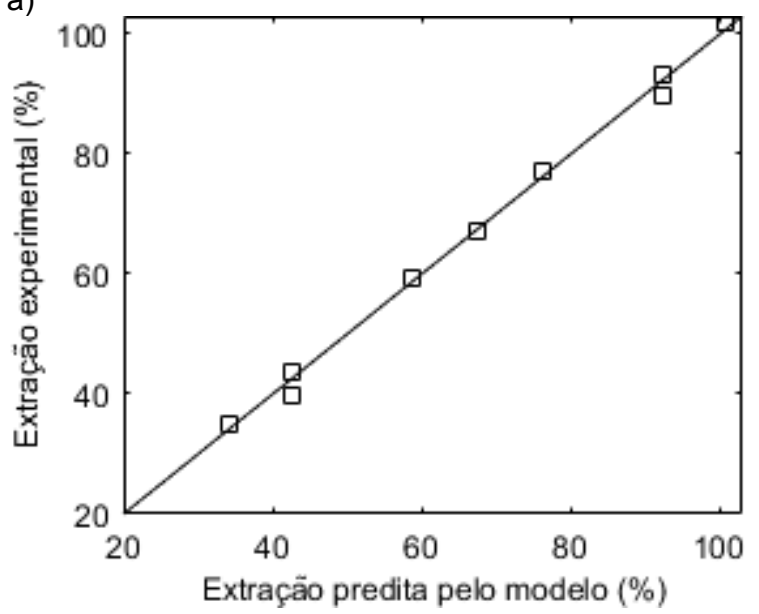

Em âmbito industrial, entretanto, pode não ser viável - tanto técnica quanto economicamente - operar o processo nas condições ótimas reportadas pelo modelo. Isso se deve ao fato de a condição ótima empregar temperatura, pureza e quantidade de solvente elevada. Para tanto, necessitaria maiores quantidades de etanol, de característica anidra, bem como maiores demandas de energia. Tendo isso, a partir do modelo foram realizadas simulações com o intuito de se observar regiões das variáveis estudadas que apresentam elevadas eficiências de extração no equilíbrio, conforme apresentado na Figura 2 através das curvas de níveis para a extração.

A Figura 2a aponta que ao empregar etanol hidratado com pureza de 95,5 wt\%, a maior extração percentual é de 92,5\%, empregando os maiores níveis de temperatura e razão etanol:óleo. Para etanol 97,5 wt\%, a máxima extração é de 96,5\%, conforme apontado na Figura 2b. Já na Figura 2c, com o emprego de etanol 99,5 wt $\%$, pode-se observar que em temperaturas superiores a $60^{\circ} \mathrm{C}$ e razões etanol:óleo superiores a 19:1 é possível atingir eficiências de extração maiores que $99 \%$. De forma geral, percebe-se que o teor de água presente no solvente limita a extração de óleo de soja. Rao et al. (1955), ao estudarem a solubilidade de diferentes óleos vegetais em etanol anidro e hidratado, apontaram que b)

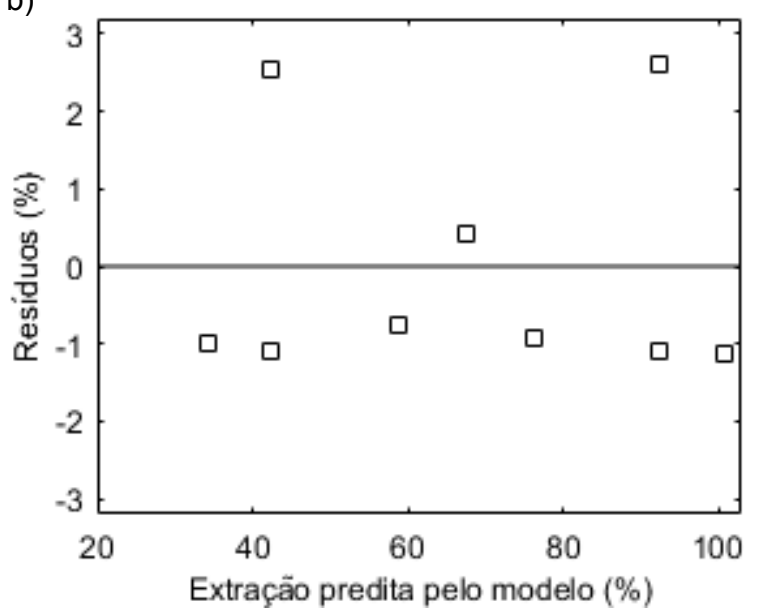

o óleo de soja e o etanol 95,5 wt\% são completamente miscíveis apenas em temperaturas maiores que $90^{\circ} \mathrm{C}$, fator não englobado nesse estudo, limitando portanto o rendimento da extração. Esse efeito também foi observado por Gandhi et al. (2003) ao estudarem a extração de óleo de soja com etanol em diferentes graus de hidratação (80 wt\%, 90 wt\%, 95,6 wt\% e anidro), reportando rendimentos maiores de extração conforme menor grau de hidratação do etanol. Dagostin et al. (2015) aponta que esse comportamento pode estar atrelado ao fato de que a incorporação de água no solvente reduz drasticamente a solubilização do óleo na mistura e, consequentemente, suprime a extração do óleo pelo solvente.

A Figura 2d, por sua vez, apresenta que se a temperatura for de $70^{\circ} \mathrm{C}$, para se atingir $99 \%$ de eficiência de extração é necessário o uso de etanol com pureza mínima de 98,8\% wt\% e uma razão etanol:óleo de 20:1. Com a utilização do solvente na forma anidra, 99,5 wt\%, pode-se atingir $99 \%$ de eficiência de extração utilizando razões etanol:óleo menores, de no mínimo 18:1, para a mesma temperatura. De acordo com Taketa et al. (2013), a utilização de solvente em excesso no processo de extração pode favorecer a etapa seguinte, a produção de biodiesel, justificando-se tal fato pelo deslocamento do equilíbrio no sentido da formação de biodiesel. 


\section{REA - Revista de estudos ambientais (Online) v.19, n. 1, p.27-35, jan./jun. 2017}

Figura 2 - Extração percentual em função da interação entre os diferentes parâmetros adotados: a) interação de T e R com P (-1; 95,5 wt\%), b) interação de T e R com P (0; 97,5 wt\%), c) interação de T e $R$ com $P\left(+1 ; 99,5\right.$ wt\%) e d) interação de $R$ e $P$ com $T\left(+1 ; 70^{\circ} \mathrm{C}\right)$

a)

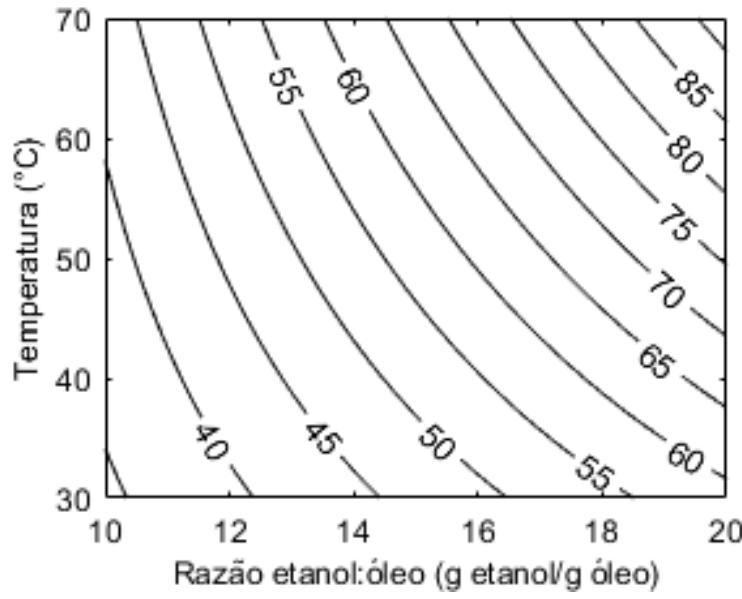

c)

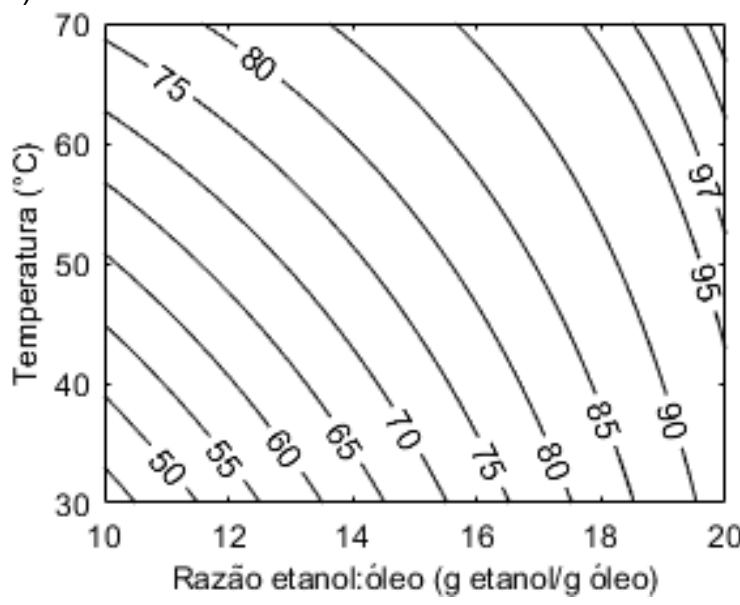

\section{Conclusões}

Neste estudo, a análise estatística por meio de planejamento experimental fatorial $2^{3}$ apontou que temperatura (entre 30 e $\left.70^{\circ} \mathrm{C}\right)$, razão etanol:óleo (10:1 à 20:1 g etanol/ óleo) e pureza do solvente (entre 95,5 e 99,5 wt\%) são variáveis que influenciam significativamente o equilíbrio do processo de extração de óleo de soja com etanol. A interação entre esses fatores não foi significativa, exceto o efeito de maior ordem. O teste de curvatura quadrática mostrou que um modelo linear de primeira ordem é suficiente para descrever os dados experimentais. A partir do modelo obtido por regressão, as condições de máxima extração no equilíbrio foram apontadas como sendo nos máximos valores das variáveis b)

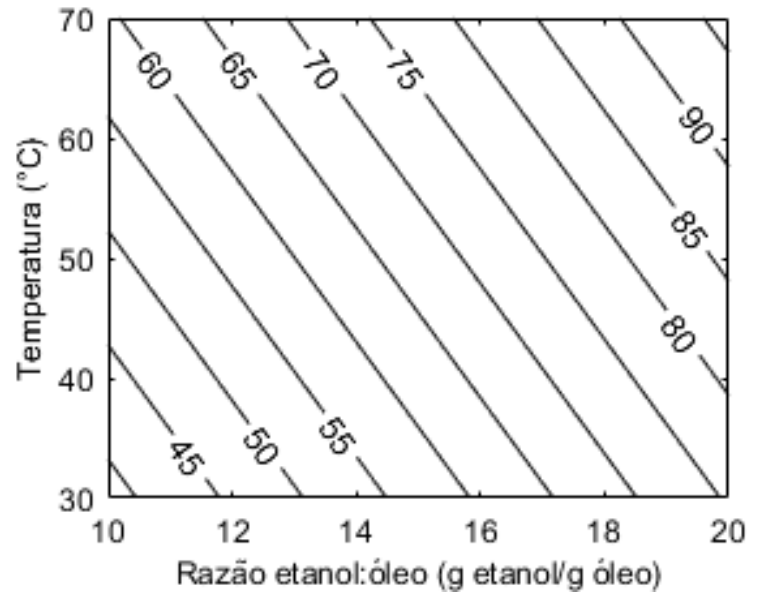

d)

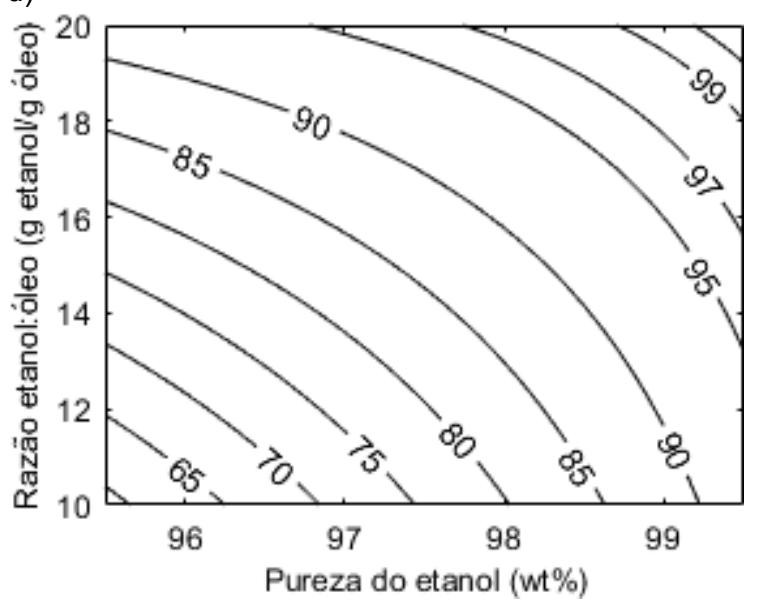

estudadas: temperatura de $70{ }^{\circ} \mathrm{C}$, razão etanol:óleo de 20:1 e pureza do solvente (etanol) de 99,5\%. Foram apresentadas também, curvas de níveis para auxiliar na seleção de combinações das variáveis que apresentam elevadas eficiências de extração. A partir dos resultados obtidos, percebeu-se que o teor de água presente no solvente limita a extração uma vez que a água no solvente reduz drasticamente a solubilização do óleo na mistura. Ainda, constatou-se que a utilização de maiores razões etanol:óleo pode ser favorável para uma posterior produção de biodiesel. Com base nisso, pode-se afirmar que o etanol é uma alternativa viável para a substituição do hexano em processos de extração de óleo de soja. 


\title{
REA - Revista de estudos ambientais (Online) v.19, n. 1, p.27-35, jan./jun. 2017
}

\author{
5 Evaluation of the Extraction of Soybean Oil with Ethanol
}

\begin{abstract}
Awareness of energy and environmental issues arising from the burning of fossil fuels has encouraged the search for renewable fuels, among them biodiesel. Industrially, it is produced via a transesterification reaction of an oil previously extracted from the oleaginous (usually the hexane as solvent), and an alcohol. In this context, it is important to evaluate the possibility of using ethanol as an extracting agent, since it also participates in the reaction, thus simplifying the process. In this work, based on an experimental design, the efficiency of the soybean seeds oil extraction, at equilibrium, using ethanol as solvent, was evaluated according to the following variables: temperature (between 30 and $70^{\circ} \mathrm{C}$ ), solvent ratio: oil 10 to $20 \mathrm{~g}$ ethanol / $\mathrm{g}$ oil) and ethanol purity (95.5 to $99.5 \mathrm{wt} \%$ ). Soybeans with a moisture and volatile content of $5.71 \mathrm{wt} \%$ and oil content of $18.31 \mathrm{wt} \%$ were used. The experiments showed extraction between $35 \%$ and $100 \%$. An analysis of variance showed that both investigated variables directly and significantly influence the extraction equilibrium. A linear first order model was sufficient to predict the experimental results and was used to calculate values of the variables that showed high extraction efficiencies.
\end{abstract}

Keywords: Solid-liquid extraction. Ethanol. Soybean oil. Experimental planning.

\section{Referências}

ALBRECHT, L. P.; BRACCINI, A. de L.; ÁVILA, M. R.; SUZUKI, L. S.; SCAPIM, C. A.; BARBOSA, M. C. Teores de óleo, proteínas e produtividade de soja em função da antecipação da semeadura na região oeste do Paraná. Bragantia, v. 67, n. 4, p. 865-873, 2008.

AMERICAN OIL CHEMISTS' SOCIETY - AOCS. Official Method Ac 2-41 -Moisture and Volatile Matter. Illinois, 3p., 2009.

AMERICAN OIL CHEMISTS' SOCIETY - AOCS. Official Method Ac 3-44 - Soybeans - Oil Content. Illinois, 3p., 1997.

ANG, G. T.; OOI, S. N.; TAN, K. T.; LEE, K. T.; MOHAMED, A. R. Optimization and kinetic studies of sea mango (Cerbera odollam) oil for biodiesel production via supercritical reaction. Energy Conversion and Management, v. 99, p. 242251, 2015.

ATABANI, A. E.; SILITONGA, A. S.; BADRUDDIN, I. A.; MAHLIA, T. M. I.; MASJUKI, H. H.; MEKHILEF, S. A comprehensive review on biodiesel as an alternative energy resource and its characteristics. Renewable and Sustainable Energy Reviews, v. 16, n. 4, p. 2070-2093, maio 2012.

BAÜMLER, E. R.; CARRÍN, M. E.; CARELLI, A. A. Extraction of sunflower oil using ethanol as solvent. Journal of Food Engineering, v. 178, p. 190-197, jun. 2016.

CAVALCANTE, A. K.; DE SOUSA, L. B.; HAMAWAKI, O. T. Determinação e avaliação do teor de óleo em sementes de soja pelos métodos de ressonância magnética nuclear e soxhlet. Bioscience Journal, v. 27, p. 8-15, 2011.
DAGOSTIN, J.; CARPINE, D.; RAMOS, L.; MAFRA, M.; CORAZZA, M. Equilíbrio líquidolíquido do sistema pseudobinário contendo óleo de soja+(etanol+ água) frente a diversas temperaturas. Blucher Chemical Engineering Proceedings, v. 1, n. 2, p. 15053-15059, 2015.

DAGOSTIN, J. L. A.; CARPINÉ, D.; CORAZZA, M. L. Extraction of soybean oil using ethanol and mixtures with alkyl esters (biodiesel) as cosolvent: Kinetics and thermodynamics. Industrial Crops and Products, v. 74, p. 69-75, 2015.

FERNÁNDEZ, C. M.; FIORI, L.; RAMOS, M. J.; PÉREZ, Á.; RODRÍGUEZ, J. F. Supercritical extraction and fractionation of Jatropha curcas $L$. oil for biodiesel production. The Journal of Supercritical Fluids, v. 97, p. 100-106, 2015

FERREIRA-DIAS, S.; VALENTE, D. G.; ABREU, J. M. F. Comparison between ethanol and hexane for oil extraction from Quercus suber $\mathrm{L}$. fruits. Grasas y Aceites, v. 54, n. 4, p. 378-383, 30 dez. 2003.

FREITAS, S. P.; LAGO, R. C. A. Dados de Equilíbrio para o Processo de Extração de Óleos de Café e Girassol Usando Etanol. Brazilian Journal of Food Technology, v. 10, n. 3, p. 220224, 2007.

GANDHI, A. P.; JOSHI, K. C.; JHA, K.; PARIHAR, V. S.; SRIVASTAV, D. C.; RAGHUNADH, P.; KAWALKAR, J.; JAIN, S. K.; TRIPATHI, R. N. Studies on alternative solvents for the extraction of oil-I soybean. International Journal of Food Science and Technology, v. 38, n. 3, p. 369375, 2003.

HINCAPIÉ, G.; MONDRAGÓN, F.; LÓPEZ, D. Conventional and in situ transesterification of castor seed oil for biodiesel production. Fuel, v. 90, n. 4, p. 1618-1623, 2011. 


\section{REA - Revista de estudos ambientais (Online) v.19, n. 1, p.27-35, jan./jun. 2017}

IPCC - INTERGOVERNMENTAL PANEL ON CLIMATE CHANGE (ed.). Climate change 2014: mitigation of climate change: Working Group III contribution to the Fifth Assessment Report of the Intergovernmental Panel on Climate Change. New York, NY: Cambridge University Press, 2014.

KARTIKA, I. A.; EVON, P.; CERNY, M.; SUPARNO, O.; HERMAWAN, D.; ARIONO, D.; RIGAL, L. Simultaneous solvent extraction and transesterification of jatropha oil for biodiesel production, and potential application of the obtained cakes for binderless particleboard. Fuel, v. 181, p. $870-877,2016$.

KNOTHE, G.; VAN GERPEN, J. H.; KRAHL, J. J.; GERPEN, J. H. Van. The Biodiesel Handbook. [s.l.] AOCS Publishing, 2005. v. 2

LI, Y.; FINE, F.; FABIANO-TIXIER, A.-S.; ABERTVIAN, M.; CARRE, P.; PAGES, X.; CHEMAT, F. Evaluation of Alternative Solvents for Improvement of Oil Extraction from Rapeseeds. Comptes Rendus Chimie, v. 17, n. 3, p. 242251, mar. 2014.

MANDARINO, J. M. G.; ROESSING, A. C. Tecnologia para Produção de Óleo de Soja: Descrição das Etapas, Equipamentos, Produtos e Subprodutos. Embrapa, Londrina, PR, p. 100, 2001.

MONTGOMERY, D. C.; RUNGER, G. C. Applied Statistics and Probability for Engineers. 3. ed. New York: John Wiley \& Sons, Inc, 2003.

RAI, A.; MOHANTY, B.; BHARGAVA, R. Supercritical extraction of sunflower oil: A central composite design for extraction variables. Food Chemistry, v. 192, p. 647-659, fev. 2016.

RAO, R. K.; KRISHNA, M. G.; ZAHEER, S. H.; ARNOLD, L. K. Alcoholic extraction of vegetable oils. I. Solubilities of cottonseed, peanut, sesame, and soybean oils in aqueous ethanol. Journal of the American Oil Chemists Society, v. 32, n. 7 , p. 420-423, jul. 1955.

RENEWABLE ENERGY POLICY NETWORK FOR THE 21ST CENTURY - REN21. Renewables 2015: global status report. Paris: REN21 Secretariant, 2015.

RENEWABLE FUELS ASSOCIATION. 2016 Ethanol Industry Outlook. p. 1-31, 2016.

RODRIGUES, C. E. C. Utilização de solvente biorenovável nos processos de extração e desacidificação de óleos vegetais. 2011. Tese (Livre-Docência na área de Equilíbrio de Fases e Processos de Separação na Indústria de Alimentos) - Faculdade de Zootecnia e Engenharia de Alimentos, Universidade de São Paulo. Pirassununga, 172p, 2011.
SANGALETTI-GERHARD, N.; ROMANELLI, T. L.; VIEIRA, T. M. F. de S.; NAVIA, R.; REGITANOD'ARCE, M. A. B. Energy flow in the soybean biodiesel production chain using ethanol as solvent extraction of oil from soybeans. Biomass and Bioenergy, v. 66, p. 39-48, 2014.

SAWADA, M. M.; VENÂNCIO, L. L.; TODA, T. A.; RODRIGUES, C. E. C. Effects of different alcoholic extraction conditions on soybean oil yield, fatty acid composition and protein solubility of defatted meal. Food Research International, v. 62, p. $662-670,2014$.

TAKETA, T. B.; FERREIRA, M. Z.; GOMES, M. C. S.; PEREIRA, N. C. Influência da temperatura, razão molar (óleo de Soja/etanol) e tipo de óleo de soja na reação de transesterificação. Revista Tecnológica, v. 22, p. 75-82, 2013.

THOMAS, G. Análise teórico-experimental da extração de óleo de soja em instalação industrial do tipo Rotocell. 2003. Tese (Doutorado em Engenharia) - Programa de PósGraduação em Engenharia Mecânica, PROMEC, Escola de Engenharia, Universidade Federal do Rio Grande do Sul. Porto Alegre, 125p, 2003.

TOMAZIN JR., C. Extração de óleo de soja com etanol e transesterificação etílica na miscela. 2008. Dissertação (Mestrado em Ciências) Programa de Pós-Graduação em Ciências, Centro de Energia Nuclear na Agricultura, Universidade de São Paulo. Piracicaba, 64p, 2008.

TUNTIWIWATTANAPUN, N.; TONGCUMPOU, C.; WIESENBORN, D. Optimization of alcoholic soybean oil extraction as a step towards developing in-situ transesterification for fatty acid isopropyl esters. Industrial Crops and Products, v. 94, p. 189-196, dez. 2016.
U. S.
ENERGY
INFORMATION ADMINISTRATION. International Energy Outlook 2016. [s.l: s.n.]v. 0484

ZACHI, R. Influência da Temperatura no Processo de Extração de Óleo de Soja em Leito Fixo. 2007. Dissertação (Mestrado em Modelagem Matemática) - Departamento de Tecnologia, Departamento de Física, Estatística e Matemática, Universidade Regional do Noroeste do Estado do Rio Grande do Sul, 2007.

\section{Agradecimentos}

Os autores são gratos a Universidade Federal da Fronteira Sul (UFFS), ao Conselho Nacional de Desenvolvimento Científico e Tecnológico $(\mathrm{CNPq})$ e à Fundação de Amparo à Pesquisa do Estado do Rio Grande do Sul (FAPERGS) pelo suporte financeiro. 